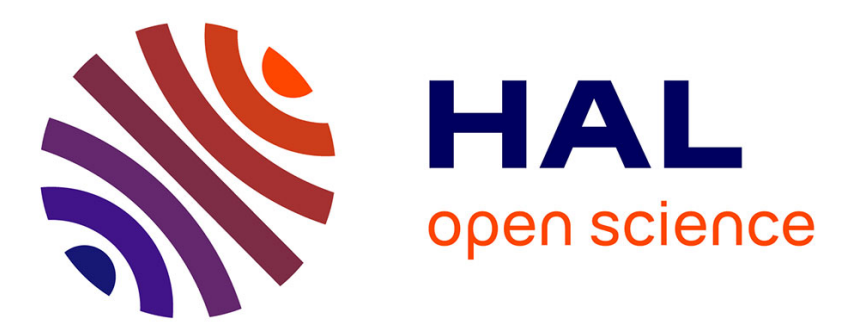

\title{
FLUCTUATIONS THERMIQUES A L'INTERFACE LIQUIDE-VAPEUR DE L'ANHYDRIDE CARBONIQUE PRÈS DU POINT CRITIQUE
}

\author{
M. Bouchiat, J. Meunier
}

\section{- To cite this version:}

M. Bouchiat, J. Meunier. FLUCTUATIONS THERMIQUES A L'INTERFACE LIQUIDE-VAPEUR DE L'ANHYDRIDE CARBONIQUE PRÈS DU POINT CRITIQUE. Journal de Physique Colloques, 1971, 32 (C5), pp.C5a-181-C5a-184. 10.1051/jphyscol:1971522 . jpa-00214742

HAL Id: jpa-00214742

https://hal.science/jpa-00214742

Submitted on 1 Jan 1971

HAL is a multi-disciplinary open access archive for the deposit and dissemination of scientific research documents, whether they are published or not. The documents may come from teaching and research institutions in France or abroad, or from public or private research centers.
L'archive ouverte pluridisciplinaire HAL, est destinée au dépôt et à la diffusion de documents scientifiques de niveau recherche, publiés ou non, émanant des établissements d'enseignement et de recherche français ou étrangers, des laboratoires publics ou privés. 


\title{
FLUCTUATIONS THERMIQUES A LINTERFACE LIQUIDE-VAPEUR DE L'ANHYDRIDE CARBONIQUE PRẼS DU POINT CRITIQUE
}

\author{
M. A. BOUCHIAT et J. MEUNIER \\ Laboratoire de Spectroscopie Hertzienne de l'E. N. S. associé au C. N. R. S. \\ Paris, France
}

\begin{abstract}
Résumé. - L'analyse spectrale de la lumière diffusée par un interface permet de mesurer la tension superficielle et la viscosité du fluide sans perturber l'état d'équilibre. On indique la méthode du calcul théorique de la forme spectrale exacte de la lumière diffusée. On décrit le montage expérimental utilisé pour l'interface liquide vapeur de $\mathrm{CO}_{2}$ près du point critique et l'on compare les spectres expérimentaux et théoriques.
\end{abstract}

Abstract. - The spectrum analysis of light scattered from thermally excited surface waves on a liquid interface allows the measurement of the surface tension and the viscosity without disturbing thermal equilibrium. The method used to obtain the theoretical line shape is briefly outlined. The liquid-vapor interface of Carbon dioxide has been studied. The experimental arrangement is described. The experimental and theoretical spectrum are compared.

Sur un interface liquide l'agitation thermique produit des fluctuations qui peuvent se propager sous forme d'ondes de capillarité ou de gravité amorties par la viscosité. Ces ondes, les "phonons de surface " diffusent un faisceau incident de lumière cohérente avec un léger changement de fréquence (quelques dizaines à quelques milliers de $\mathrm{Hz}$ ) détectable par spectroscopie de battements lumineux [1]. Cet effet représente pour la surface d'un liquide l'analogue de l'effet Brillouin pour la diffusion en volume. L'analyse spectrale de la lumière diffusée donne par simple translation de fréquence le spectre des fluctuations de la coordonnée verticale des points de l'interface et permet d'obtenir la tension superficielle $\alpha$ ou la viscosité $\eta$ d'un liquide (ou les deux paramètres à la fois) sans perturber l'état d'équilibre. Cette méthode offre un intérêt tout particulier au voisinage d'un point critique où l'interface disparaît, les méthodes de mesure classiques devenant alors inadéquates. Or pour la vérification des lois d'échelle il est important de bien connaître la variation de $\alpha$ et de $\eta$ avec la température dans la «zone critique », c'est-àdire assez près de $T_{\mathrm{c}}$ pour que la portée des corrélations spatiales devienne grande devant les distances caractéristiques du potentiel intermoléculaire. Nous décrivons ici quelques résultats concernant l'interface liquide-vapeur de $\mathrm{CO}_{2}$ près de $T_{\mathrm{c}}$. Auparavant nous donnons de brèves indications sur le spectre théorique.

1) Répartition spectrale de la lumière diffusée. Nous avons calculé le spectre des fluctuations thermiques de la coordonnée verticale $\zeta(\mathrm{r}, t)$ des points de la surface libre d'un liquide [2] en adaptant à ce problème les hypothèses utilisées par Landau et Placzek et reprises par Mountain [3], pour l'étude des fluctuations de densité en volume : étant donné l'échelle macroscopique de dimension et de temps caractéristique des fluctuations qui nous intéressent, on peut raisonnablement décrire leur évolution à l'aide des équations de l'hydrodynamique classique; de plus on peut linéariser ces équations puisqu'il s'agit de déplacements ayant une amplitude très faible devant leur longueur d'onde. Les modes de surface correspondants à des valeurs de q différentes peuvent être considérés comme indépendants; nous allons discuter dans la suite l'évolution d'un mode de vecteur d'onde q fixé correspondant à une déformation du type $\zeta_{\mathbf{q}}(t)$ eiq.r. Si à l'instant $t=0$, $\zeta_{q}(0)$ n'est pas nul (du fait de l'action d'une force extérieure), les équations de l'hydrodynamique permettent de calculer $\zeta_{\mathbf{q}}(\tau)$ à tout instant ultérieur pourvu que l'état initial soit complètement spécifié. On démontre qu'il en est bien ainsi lorsqu'on se fixe à $t=0$ la valeur de $\zeta_{\mathbf{q}}$ et de $v_{z, \mathbf{q}}$ (la composante verticale de la vitesse du fluide en tout point $z)$. $\zeta_{\mathbf{q}}(\tau)$ est alors donné par une expression de la forme :

$$
\zeta_{\mathbf{q}}(\tau)=\zeta_{\mathbf{q}}(0) f_{q}(\tau)+\int_{-\infty}^{+\infty} v_{z, \mathbf{q}}(z, 0) g_{q}(z, \tau) \mathrm{d} z
$$

linéaire par rapport aux conditions initiales.

Considérons maintenant le problème des fluctuations. Envisageons parmi un ensemble de systèmes identiques du point de vue thermodynamique, tous ceux qui à l'instant $t=0$ présentent des fluctuations de mode $q$, identiques. On admettra que l'équation (1) décrit l'évolution moyenne de ces systèmes particuliers, c'est-à-dire qu'elle donne la valeur moyenne de $\zeta_{\mathbf{q}}(\tau)$ (pour $\tau>0$ ). Si maintenant on considère l'ensemble complet de systèmes figuratif de l'état d'équilibre, 
$\zeta_{q}(0)$ et $v_{z q}(z, 0)$ varient aléatoirement de l'un à l'autre mais $f(\tau)$ et $g(z, \tau)$ demeurent des fonctions certaines de $\tau$; ce qui conduit à l'écrire :

$$
\begin{aligned}
& \overline{\zeta_{\mathbf{q}}(0) \zeta_{\mathbf{q}}^{*}(\tau)}=\overline{\left|\zeta_{\mathbf{q}}(0)\right|^{2}} J_{q}^{*}(\tau)+ \\
& \quad+\int_{-\infty}^{+\infty} \overline{\zeta_{\mathbf{q}}(0) v_{z \mathbf{q}}^{*}(z, 0)} g_{q}^{*}(z, \tau) \mathrm{d} z \text { pour } \tau>0
\end{aligned}
$$

(l'expression pour $\tau$ quelconque s'obtient simplement en remarquant que c'est une fonction paire de $\tau$ ). Les valeurs moyennes qui figurent dans (2) s'évaluent en appliquant les principes de la mécanique statistique à un système à l'équilibre :

$$
\overline{\zeta_{\mathbf{q}}(0) v_{z, \mathbf{q}}^{*}(z, 0)}=0, \overline{\left.\zeta_{\mathbf{q}}(0)\right|^{2}}=k T / \alpha q^{2} .
$$

La première relation résulte de l'invariance par rapport au sens du temps du hamiltonien du système microscopique.

On voit que finalement la fonction de corrélation ou encore la puissance spectrale des fluctuations $P_{q}^{1}(\omega)$, s'obtient directement à partir de $f_{q}(\tau)$ c'est-à-dire de la

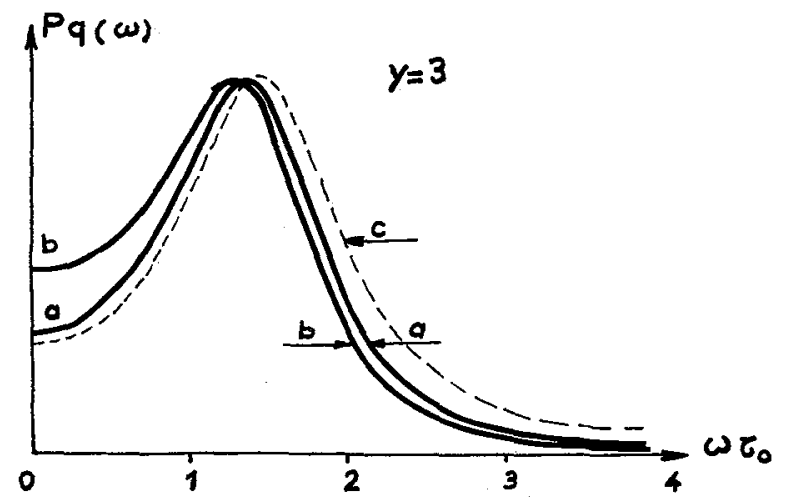

(a)

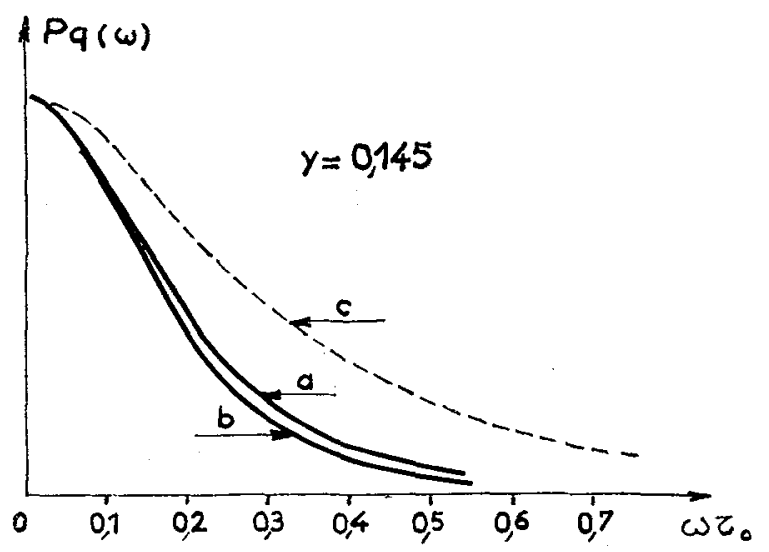

(b)

FIG. 1. - Spectre théorique des fluctuations thermiques de la surface libre $P_{1}(\omega)$ (Courbe $a$ ), spectre des fiuctuations de la coordonnée d'un oscillateur harmonique $P_{2}(\omega)$ (Courbe $b$ ), et spectre correspondant à la superposition de 2 courbes de Lorentz (Courbe $c$ ), construits pour la même valeur du paramètre $y=\alpha \rho / 4 \eta^{2} q$, en unités réduites de pulsation, $\tau_{0}=\rho / 2 \eta q^{2}$. solution du problème hydrodynamique correspondant aux conditions initiales $\zeta_{\mathbf{q}}(0)$ fixé, $v_{z, q}(z, 0)=0$.

Il est intéressant de noter sur le résultat - que nous ne donnerons pas ici - que l'excitation thermique d'un mode présente une assez grande analogie avec celle d'un oscillateur harmonique à une dimension, spécialement dans le cas d'un amortissement très fort (forte viscosité) ou très faible (faible viscosité). Cependant dans une étroite zone d'amortissement interdiaire $P_{q}^{1}(\omega)$ diffère de manière appréciable du spectre des fluctuations thermiques d'un oscillateur harmonique $P_{q}^{2}(\omega)$, comme le montre la figure 1 . On peut interpréter ceciểde la manière suivante : lorsque l'interface liquide se déplace le mouvement ne se transmet pas instantanément aux couches fluides voisines, et au retard correspondant est associée une dissipation d'énergie. Il est normal de constater que la différence entre $P_{q}^{1}(\omega)$ et $P_{q}^{2}(\omega)$ est maximum lorsque le retard est de l'ordre du temps d'évolution propre des ondes de surface.

Dans le cas de l'interface liquide-vapeur de $\mathrm{CO}_{2}$ près du point critique il est assez important de connaître la forme spectrale exacte. Très loin de $T_{\mathrm{c}}$ les ondes de surface sont très peu amorties car la viscosité du fluide est très faible. Lorsqu'on se rapproche de $T_{\mathrm{c}}$ la tension superficielle qui est à l'origine de la force de rappel décroît rapidement (comme $\left.\left(T_{\mathrm{c}}-T\right)^{1,25}\right)$, sans que la viscosité subisse d'importante variation; ceci entraîne un amortissement de plus en plus fort et vers

$$
T_{\mathrm{c}}-T \approx 10^{-2}{ }^{\circ} \mathrm{C}
$$

on arrive dans la zone où la différence entre $P_{q}^{1}$ et $P_{q}^{2}$ n'est plus négligeable pour certaines valeurs de $q$.

2) Schéma de l'expérience. - Une première série d'expériences a été réalisée en éclairant la surface sous incidence normale [4]. La mesure de la constante de capillarité $\alpha$ dans le domaine de température

$$
30 \geqslant T_{\mathrm{c}}-T \geqslant 0,04{ }^{\circ} \mathrm{C}
$$

nous a conduits à :

$$
\mid \begin{aligned}
\alpha & =\alpha_{0}\left[\frac{T_{\mathrm{c}}-\mathrm{T}}{T_{\mathrm{c}}}\right]^{\mu} \\
\alpha_{0} & =77 \pm 5 \text { dynes } / \mathrm{cm} \\
\mu & =1,253 \pm 0,010 .
\end{aligned}
$$

Nous avons pu d'autre part faire une mesure de viscosité très près de $T_{\mathrm{c}}$ mais avec une faible précision : $T_{\mathrm{c}}-T=0,034{ }^{\circ} \mathrm{C}, \eta=0,35 \pm 0,05 \times 10^{-3}$ poise.

Nous allons décrire un nouveau montage qui nous permet d'améliorer beaucoup le rapport signal sur bruit. Il utilise pour angle d'incidence l'angle limite en éclairant l'interface du côté de plus fort indice. Cet angle varie beaucoup avec $T_{\mathrm{c}}-T$ et croît lorsqu'on approche de $T_{\mathrm{c}}$. Le rendement de la diffusion se trouve 
considérablement accru pour cette incidence (Fig. 2). Ce gain d'intensité est d'autant plus grand que l'on est près de $T_{\mathrm{c}}$ (un facteur $100 \mathrm{à} 0,01^{\circ} \mathrm{C}$ de $T_{\mathrm{c}}$ ) (Fig. 3) ce qui est fort important puisque la diffusion en volume tend à l'emporter sur la diffusion en surface à l'approche du point critique.

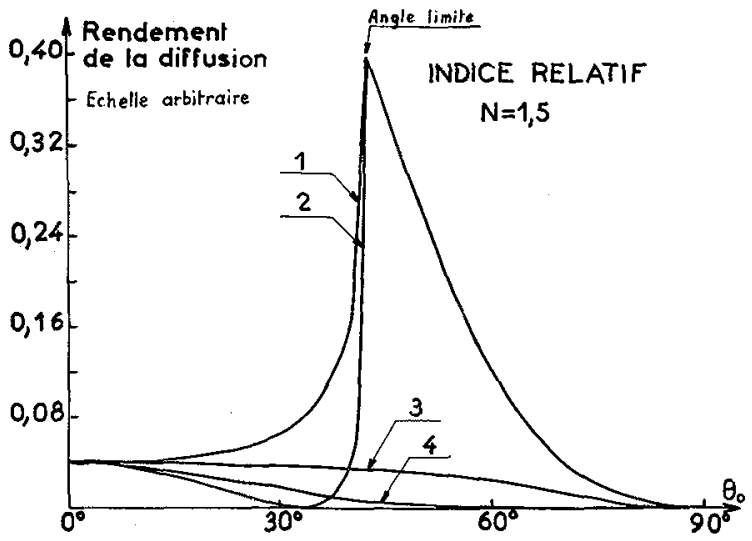

Fig. 2. - Rendement $R$ de la diffusion en fonction de l'angle d'incidence $\theta_{0}$. Courbes 1 et 2 , l'interface est éclairé du côté du plus fort indice avec une polarisation // ou $\perp$ au plan d'incidence. Courbes 3 et 4 mêmes données pour le même interface éclairé de l'autre côté.

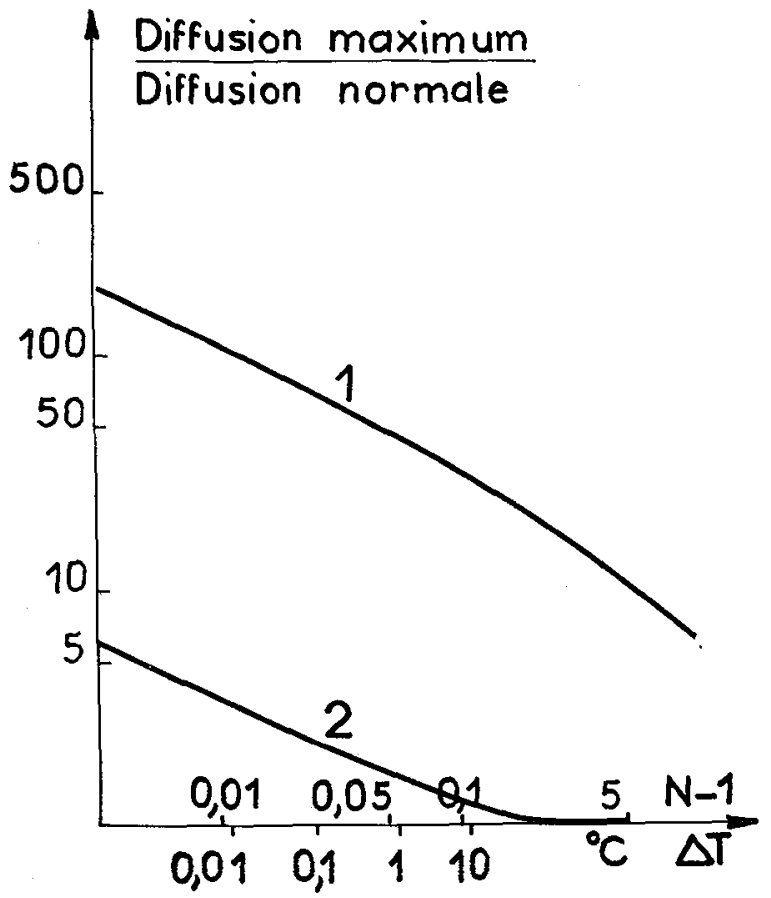

FIG. 3. - Variation en fonction de l'indice relatif $N$, du rapport entre les rendements de la diffusion sous incidence optimum et sous incidence normale. Courbes 1 et 2 pour l'interface éclairé du côté du plus fort ou du plus faible indice.

Le montage utilise comme source lumineuse un laser $\mathrm{He}-\mathrm{Ne}$ de $0,1 \mathrm{~mW}$. Le vecteur d'onde des fluctuations de surface étudiées est fixé par la position du diaphragme $\mathrm{D}_{2}$ (Fig. 4) qui est mobile sur un axe. horizontal normal au faisceau lumineux réfléchi. Dans ces conditions le nombre d'onde $q$ des fluctuations et l'angle $\varphi$ entre le faisceau réfléchi sur l'interface et le faisceau diffusé sont liés par une relation très simple $q=\frac{2 \pi}{\lambda} \varphi(\lambda=6328 \AA)$ qui reste valable même en présence des très forts gradients de densité du fluide près de $T_{c}$. La cellule contenant l'anhydride carbonique est placée dans un thermostat : les fluctuations résiduelles de température sont de $2 \times 10^{-4}{ }^{\circ} \mathrm{C}$ sur le temps de mesure d'un spectre.

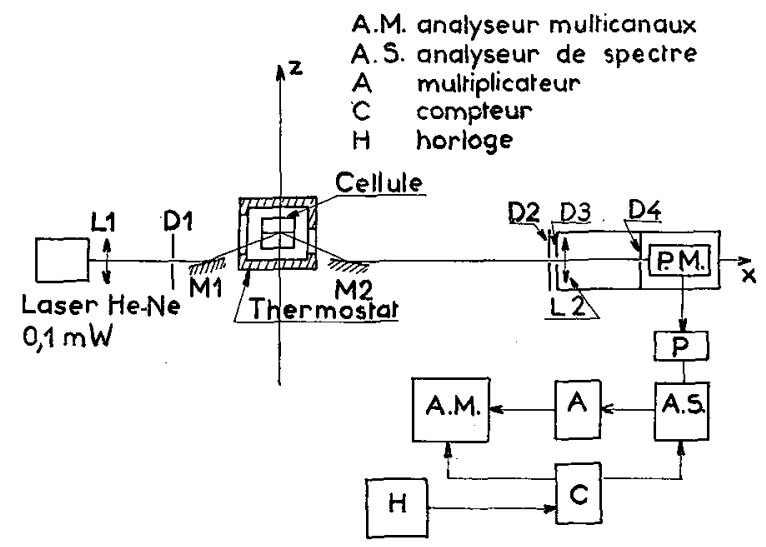

Fig. 4. - Schéma du montage expérimental.

Le photomultiplicateur P. M. reçoit la lumière diffusée par l'interface et la lumière diffusée par les imperfections des fenêtres de la cellule, qui joue le rôle d'oscillateur local ; les intensités de ces deux faisceaux sont comparables si bien que la détection par battements lumineux est assez complexe : le signal est la somme d'un spectre hétérodyne et d'un spectre homodyne d'intensités comparables. La figure 5 montre un exemple de spectre obtenu sur ce montage : les points sont expérimentaux, la courbe en trait plein et la

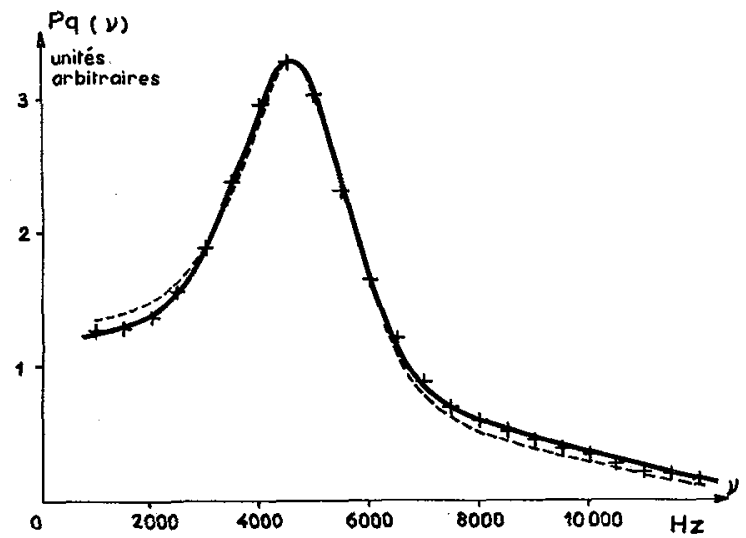

Fig. 5. - Etude spectrale de la lumière diffusée par l'interface liquide vapeur de $\mathrm{CO}_{2}$ à $T_{\mathrm{c}}-T=0,59^{\circ} \mathrm{C}, q=3325 \mathrm{~cm}^{-1}$. Les points sont expérimentaux, les 2 courbes sont théoriques : en plein $P_{1}(\omega)$, en pointillé $P_{2}(\omega)$.

On en déduit $\alpha=3,32 \times 10^{-2}$ dynes $/ \mathrm{cm}, \eta=0,37 \times 10^{-3}$ poise. L'élargissement instrumental est totalement négligeable. 
courbe en pointillés correspondent respectivement aux spectres $P_{1}(v)$ et $P_{2}(v)$ tels que l'écart quadratique moyen avec les points expérimentaux soit minimum. Ces résultats montrent que le spectre des fluctuations de surface diffère du spectre des fluctuations d'un oscillateur harmonique dans la zone d'amortissement critique, comme permet de le prévoir la théorie.
Conclusion. - Grâce à l'amélioration considérable du rapport signal sur bruit, le domaine des fluctuations accessibles $\left(200\right.$ à $\left.8000 \mathrm{~cm}^{-1}\right)$ est élargi et la résolution spectrale accrue. Ceci doit nous permettre d'une part d'approcher plus près du point critique, d'autre part de mesurer de manière plus précise la tension superficielle et surtout la viscosité.

\section{Bibliographie}

[1] Voir par exemple Bouchiat (M. A.) et Meunier (J.). Polarisation, Matière et Rayonnement (P. U.F. Paris, 1969), p. 121, où d'autres références sont données.
[2] Bouchiat (M. A.) et Meunier (J.), J. Physique 1971, $32,561$.

[3] Mountain (R. D.), Rev. Mod. Phys, 1966, 38, 205.

[4] Meunier (J.), J. Physique, 1969, 30, 933. 